\title{
FATORES NUTRICIONAIS E FÍSICOS QUE INTERFEREM NA QUALIDADE DE VIDA DO IDOSO
}

ANDREOTTI, Daniela Galassi. Bacharel em Nutrição - UNIARA. E-mail: Daniela.andreotti@ hotmail.com VEIGA, Maria Leonor Ruiz. Bacharel e Licenciada em Educação Física - UNIARA. E-mail:noveiga@ hotmail.com.br.

MORAES, Marina Miguel Giovanelli de. Bacharel em Nutrição - UNIFIA. Email:moraesmarina@yahoo.com.br.

BEGNAMI, Maristela de Fátima Damião. Bacharel em Nutrição/Faculdade Santa Giulia. E-mail: maristela_damiao@hotmail.com.

COGLIATTI, Wilson Nicola Júnior. Bacharel e Licenciado em Educação Física - UNIP. E-mail: coglia@terra.com.br.

PEREIRA, Rita de Cássia Garcia. Nutricionista/Docente do curso de Nutrição - UNIARA. E-mail: ritanutry@terra.com.br.

\section{Resumo}

O envelhecimento populacional é uma realidade brasileira e motivo de desafio para a saúde pública, visto que no envelhecimento ocorrem alterações funcionais inerentes ao processo de envelhecer, as quais devem ser consideradas a fim de proporcionar ao idoso qualidade de vida, de forma que o mesmo possa passar por esse processo com saúde. Este artigo de revisão bibliográfica se propõe a discutir a questão do envelhecimento sob a ótica da nutrição e da atividade física, destacando os principais fatores nutricionais e físicos que afetam a qualidade de vida do ser humano durante o envelhecimento, em razão da elevação da expectativa de vida da população e do crescente aumento da participação do idoso na sociedade, principalmente nos países em desenvolvimento como o Brasil. Por meio do conhecimento de tais causas e da intervenção de nutricionistas e educadores físicos, tal população poderá ter alimentação adequada às suas necessidades, treinamentos físicos moldados às condições do seu corpo e, consequentemente, mais saúde e independência para continuar desempenhando as atividades do dia-a-dia.

Palavras-chave: Idoso; Nutrição; Atividade física.

\section{NUTRITIONAL AND PHYSICAL FACTORS THAT INTERFERE IN THE QUALITY OF LIFE OF ELDERLY PEOPLE}

\begin{abstract}
Population aging is a Brazilian reality and a challenging aspect for public health since functional changes that are inherent to such process occur which should be taken into consideration in order to provide the elderly with good quality of life so that they can go through this process healthly. This review article aims todiscuss the issue of aging from the perspective of nutrition and physical activity, highlighting the main nutrition and physical factors that affect the quality of human life during the aging process considering the raise in life expectancy and the increasing participation of the elderly in society, especially in developing countries like Brazil. The knowledge of these causes and the intervention of nutritionists and fitness trainers will allow such population to have its dietary needs met according to its needs, physical training tailored to the conditions of their bodies and, consequently, better health and independence to continue performing day-to-day activities.
\end{abstract}

KEYWORDS: Elderly; Nutrition; Physical activity. 


\section{Envelhecimento Populacional}

O Brasil apresenta índices de envelhecimento superiores aos dos países desenvolvidos, sendo, por esse motivo, um destaque de âmbito mundial, quando se trata desse assunto (WONG; CARVALHO, 2006). Estimativas demonstram que, no ano de 2020, o Brasil terá uma população idosa acima de 30 milhões de pessoas, ocupando a sexta posição neste ranking global (CARVALHO; GARCIA, 2003).

Nos países desenvolvidos, no final da década de 1940 e início de 1950, ocorreram alguns fatores decisivos para o prolongamento da vida, os quais deram início ao fenômeno de envelhecimento populacional, tais como: queda da mortalidade devido a estudos e avanços da área médica, redução de doenças contagiosas e parasitárias, resultado da urbanização adequada e melhorias sanitárias das cidades, elevação do nível de higiene pessoal ocasionado pelo aumento da escolaridade, melhoria nutricional decorrente de salários mais dignos e aquisição de alimentos antes inacessíveis (MENDES; GUSMÃO; FARO, 2005). Outro fator causador do envelhecimento populacional, e, como o motivador de tal processo, é a queda da fecundidade, que em 2010 foi de 1,86 filho por mulher no país; em 2000, essa taxa era de 2,38 (CARVALHO; GARCIA, 2003; IBGE, 2012). Segundo Fernandes et al. (2011), a causa dessa queda da fecundidade vem acontecendo desde o final dos anos 1970, devido à crescente participação da mulher no mercado de trabalho formal e ao uso de contraceptivos.

O Censo 2010 demonstra que, além da queda da fecundidade, a participação da população em idade ativa (15 a 64 anos de idade) teve um aumento de $54,6 \%$ para $68,5 \%$ da população total. Já o aumento na participação da população de 65 anos ou mais, nas últimas quatro décadas, saltou de $2,7 \%$ para $7,4 \%$ (IBGE, 2012).

\section{Alterações fiSiológiCas no enVelHeCimento}

Muitos fatores naturais modificam a vida dos idosos. Entre eles, destacam-se fatores físicos, fisiológicos, metabólicos, patológicos, psicológicos, sociais, culturais e econômicos, que, na maioria das vezes, comprometem o estado nutricional e a qualidade de vida desses indivíduos (BARRETO, 2004).

No envelhecimento ocorre declínio progressivo de todos os processos fisiológicos (NÓBREGA et al., 1999), e a qualidade de vida agregada a tal processo pode ser considerada uma conquista da sociedade, já que o prolongamento da existência vem sendo buscado pela humanidade desde seus primórdios (VERAS, 2009). Todos os órgãos e sistemas do corpo envelhecido sofrem várias alterações funcionais, dentre elas a redução da massa magra, o aumento do tecido adiposo e a menor eficiência de bombeamento do coração, podendo acarretar diminuição do fluxo sanguíneo. Outras alterações inerentes ao envelhecimento são: diminuição do olfato e do paladar, o que prejudica o apetite do idoso, bem como a mastigação dificultada em razão da perda dos dentes e diminuição da salivação; a constipação, resultado da secreção ineficiente de ácido clorídrico e bile, o que leva à perda do tônus gastrointestinal (MENEZES; MARUCCI, 2005). Com o passar dos anos, o corpo começa a apresentar naturalmente algumas mudanças que podem interferir na alimentação do idoso (PIERINI, NICOLA, OLIVEIRA, 2009).

\section{Alterações na capacidade sensorial e mastigatória}

As alterações sensoriais ocorrem em proporções individualizadas; as disfunções no paladar e odor tendem a começar nos 60 anos de idade, tornando-se mais severas aos 70 anos, quando diminui a habilidade de reconhecer os gostos - doce, salgado, azedo, amargo -, induzindo, assim, as mudanças metabólicas e reduzindo as secreções salivares (MAHAN, SCOTT-STUMP, 2001). Na terceira idade, um dos fatores mais relevantes na diminuição do consumo alimentar é a diminuição da sensibilidade por gostos primários doce, amargo, ácido e salgado (MORIGUTI, IUCIF, FERRIOLI, 1998).

Algumas alterações na capacidade mastigatória do idoso são decorrentes do aparecimento frequente de cáries e doenças periodontais; das próteses totais ou parciais inadequadas ou em péssimo estado de conservação; e da ausência de dentes, o que interfere 
no comportamento inicial do processo digestivo, favorecendo sua inadequação tanto no aspecto enzimático como no mecânico (CAMPOS, MONTEIRO, ORNELAS, 2000). Indivíduos que usam dentaduras e ou próteses mastigam de $75 \%$ a $85 \%$ menos eficientemente que aquelas com dentes naturais, o que leva à diminuição do consumo de carnes, frutas e vegetais frescos, ocasionando maior ingestão de alimentos macios, facilmente mastigáveis, pobres em fibras, vitaminas e minerais, fato que pode ocasionar consumo inadequado de energia por excesso, e ingestão diminuída de ferro e vitaminas.

Também podem ocorrer perdas de audição, coordenação e visão, levando à redução de ingestão de alimentos e apetite diminuído, uma vez que o reconhecimento dos alimentos se encontra alterado (MAHAN, SCOTT STUMP, 2001).

\section{Alterações na capacidade digestiva}

A atrofia da mucosa gástrica no idoso resulta na menor produção de ácido clorídrico e na diminuição na secreção do fator intrínseco, provocando uma diminuição na absorção da vitamina B12, com consequente instalação da anemia perniciosa. Esse tipo de anemia ocorre somente quando o idoso apresenta grave atrofia da mucosa gástrica. $\mathrm{O}$ estômago com gastrite leve ou moderada continua a secretar fator intrínseco suficiente para prevenir a má absorção da vitamina B12, pelo mecanismo normal (MORIGUTI, IUCIF, FERRIOLI, 1998).

\section{Alterações intestinais}

A constipação intestinal pode ser resultado da mobilidade alterada e do tônus muscular diminuído, associado à ingestão inadequada de líquidos, baixo consumo de fibras e uso aumentado de laxantes (TEIXEIRA NETO, 2003). O crescimento bacteriano excessivo no intestino pode também ocorrer, como resultado da diminuição da secreção ácida, fato que pode interferir na disponibilidade biológica dos nutrientes e pode resultar na diminuição da ação dos sais biliares, na má absorção da gordura e na diarreia (CAMPOS, MONTEIRO, ORNELAS, 2000).

\section{Diminuição da sensibilidade à sede}

Segundo o Ministério da Saúde (2010), no idoso a desidratação torna-se frequente, podendo desencadear outras doenças, como enfermidades infecciosas e cerebrovasculares.

De acordo com Campos, Monteiro, Ornelas (2000), a regulação depende da capacidade renal de concentrar e diluir a urina e a ingestão de água motivada pela sede. Deve-se considerar que a ingestão de líquidos depende dos fatores ambientais, psicológicos e fisiológicos, e que a capacidade de concentração renal diminui com a idade. Essa alteração na sensação de sede é atribuída à disfunção cerebral e ou à diminuição da sensibilidade dos osmorreceptores; contudo, a menor ingestão de líquidos pode ainda ser decorrente de alguma debilidade física, pois, neste caso, existe certa dependência de outras pessoas. O problema é chamado de hipodipsia e pode ser agravado pela administração de diuréticos e de laxantes usados muito frequentemente nos idosos, o que pode levar à desidratação.

\section{Alterações metabólicas e endócrinas}

No envelhecimento, ocorrem algumas mudanças na composição corporal, como a diminuição da massa muscular e aumento da adiposidade. Alguns autores definem tal acontecimento, quando associados a alguma limitação funcional, como sarcopneia, do grego sarco/músculo e pneia/perda (PIERINE et al., 2009).

A alteração endócrina mais frequente é a redução da produção de hormônios sexuais e hormônio do crescimento, e um aumento progressivo de concentrações de glicocorticoides e catecolamidas. Alterações na resposta insulínica ocorrem principalmente em idosos obesos (TEIXEIRA NETO, 2003).

\section{Alterações neurológicas}

Uma redução na velocidade da condução nervosa e diminuição da serotonina ocasionam estado de confusão nos idosos, que podem gerar inúmeras vezes casos de Parkinson e Alzheimer (MAHAN, SCOTTSTUMP, 2001). 


\section{Alterações cardiovasculares}

A prevalência de hipertensão arterial é crescente principalmente nas mulheres. O quadro se agrava quando o problema se associa à ação de hormônios pósmenopausa, aumento da gordura visceral, fumo, uso de bebida alcoólica, jejum prolongado, sedentarismo, antecedentes familiares, obesidade, estresse, uso de anticoncepcionais e uma dieta desequilibrada (CAETANO et al., 2008). Modificar os hábitos alimentares pode reduzir os riscos de desenvolver doenças cardiovasculares como a aterosclerose e hipertensão arterial sistêmica (MAHAN, SCOTTSTUMP, 2001).

\section{Alterações renais}

Segundo Abreu, Sesso e Ramos (1998), o peso e o volume dos rins diminuem em $20 \%$ a $30 \%$ entre as idades de 30 a 90 anos, de 250-270 g no adulto para 180-200 g no idoso. O número de glomérulos diminui em $30 \%$ a 50\%, havendo um aumento da relação entre os esclerosados e os normais: 1 em 10 glomérulos na idade de 80 anos comparados com 1 em 100 no adulto não idoso, os túbulos se mostram diminuídos em número, volume e extensão. São achados frequentes a atrofia tubular focal, fibrose intersticial e inflamação crônica, que podem estar associadas a áreas de esclerose glomerular. Acredita-se que outros fatores de risco, como doenças sistêmicas, dieta, meio ambiente e hábitos pessoais, acelerem esse processo de deterioração.

\section{Alterações respiratórias}

Para Ruivo et al. (2009), o sistema respiratório é o sistema que envelhece mais rapidamente, devido à maior exposição a poluentes ambientais ao longo dos anos. A deterioração da função pulmonar está associada ao aumento da taxa de mortalidade e, além disso, o conhecimento das mesmas contribui para a detecção e prevenção de disfunções respiratórias em idosos. Acapacidade vital (CV) sofre, geralmente, uma redução de quase $25 \%$ a $40 \%$; segundo alguns autores, a porção inicial da expiração (DEMI) permanece praticamente constante ou sofre apenas uma ligeira diminuição; o volume expiratório máximo ao primeiro segundo (VEMS) diminui claramente, a uma taxa que depende do gênero e da idade; e a razão VEMS/CVF (índice de Tiffeneau) cai progressivamente com o aumento da idade, estimando-se $73 \%$ aos 65 anos (já que o declínio da capacidade vital forçada (CVF) com a idade é um pouco menor do que o declínio do VEMS). Mais acentuadamente que qualquer outro parâmetro, a ventilação máxima voluntária (VMV) diminui.

\section{Alterações no musculoesquelético}

Segundo Ribeiro, Alves e Meira (2009), as alterações mosculoesqueléticas podem gerar dor e algum grau de dificuldade de locomoção. As articulações mais afetadas são as dos joelhos, dos pulsos, dos cotovelos e dos quadris. Na cartilagem ocorre algumas alterações, como afinamento e diminuição do líquido sinovial, prejudicando assim os ligamentos, que podem ficar mais curtos e menos flexíveis. Essas alterações resultam em menor amplitude de movimento das articulações afetadas.

Associada às alterações nas articulações, ocorre também uma perda na densidade óssea, principalmente nas mulheres pós-menopausa, podendo acarretar osteoporose. Nessa fase, pode ocorrer uma perda progressiva, absoluta, da massa óssea, até então presente como a osteopenia fisiológica (TEIXEIRA NETO, 2003).

Entre os 50 e os 70 anos, apresentam uma perda em torno de $15 \%$ por década; após a redução da força muscular, essa taxa aumenta para $30 \%$ a cada 10 anos (SOARES, 2005; MATSUDO et al., 2000). A sarcopenia contribui para outras alterações associadas à idade, como menor densidade óssea (ROSSI, 2008).

\section{Alterações na capacidade imune}

A redução da capacidade imune ocorre devido à diminuição do número de células de defesa ou disfunção, dificultando assim a habilidade em combater infecções, por apresentarem menor produção de anticorpos (MAHAN, SCOTT-STUMP, 2001). 


\section{Fatores psicossocial e socioeconômico}

Percebe-se no indivíduo que envelhece uma interação maior entre os estados psicológicos e sociais, refletidos na sua adaptação às mudanças. Uma das alterações psicossociais mais comuns é a depressão, que afeta a qualidade de vida do idoso (OLIVEIRA, 2006). Desse modo, o envelhecimento é decisivamente afetado pelo estado emocional e psicológico. Insatisfeitos principalmente pelo fato de não poderem mais trabalhar ou por isolamento interpessoal, alterações da imagem corporal e sentimento de perda, principalmente do cônjuge, são fatores que elevam as chances de depressão no idoso (BARBOSA et al., 2005).

Em alguns casos, viver sozinho pode acarretar alterações nos hábitos alimentares, em relação ao preparo, aquisição e dificuldade no preparo, devido também às habilidades motoras (MAHAN, SCOTTSTUMP, 2001).

Segundo Barbosa et al., (2005), a aposentadoria comumente causa uma crise no idoso. Com a retirada da vida de competição, a autoestima e a sensação de ser útil se reduzem. No início, a maioria dos idosos se sente satisfeita, pois parece ser muito bom poder descansar; porém, aos poucos descobrem que a vida se tornou tristemente inútil. Percebendo que ninguém necessita dele por estar isolado, recusado e excluído da sociedade, o indivíduo se sente cada vez mais angustiado, tornando difícil sua adequação ao mundo no qual vive. Além disso, a aposentadoria também acarreta uma queda no nível de renda que, por sua vez, afeta a qualidade de vida e a saúde.

O processo de envelhecimento fisiológico determina várias alterações, inclusive no aparelho locomotor, que limitam as atividades cotidianas e, assim, comprometem a qualidade de vida da pessoa que envelhece. A diminuição do nível de atividade pode levar o idoso a um estado de limitações e falta de autonomia. Evidências atuais demonstram que a atividade física traz benefícios à saúde do idoso, mantendo independência funcional e melhorando sua qualidade de vida, gerando integração social, força, equilíbrio, flexibilidade e aumento da autoestima (CRESS et al., 1999).

\section{Alimentação e envelhecimento}

A saúde do idoso depende de muitos cuidados, e a alimentação saudável está entre os mais importantes. Para Cuppari (2006), a nutrição é a ciência que estuda os alimentos, nutrientes, ação, interação e equilíbrio em relação à saúde e doença; além dos processos de ingestão, absorção, transporte, utilização e excreção de nutrientes. $\mathrm{O}$ envelhecimento promove diminuição das demandas energéticas dos idosos; tais alterações podem ser provenientes da redução metabólica e da diminuição da atividade física, devido ao avançar da idade.

Moriguti, Iucif Júnior e Ferrioli (1998) enfatizam que o envelhecimento diminui significantemente a necessidade de ingestão energética, já que a massa muscular está em declínio, resultando menos gasto calórico em repouso; a diminuição da necessidade energética é resultante da diminuição da atividade física.

Segundo o Ministério da Saúde (2006), as calorias equivalem à quantidade de energia presente em cada alimento, que vai ser disponibilizada à manutenção das funções corpóreas. A ingestão de uma alimentação desequilibrada pode levar ao fornecimento de calorias além da necessidade, ocasionando excesso de peso e consequente obesidade, ou ainda, o déficit alimentar pode causar magreza e finalmente a desnutrição.

Para Najas e Yamatto (2008), dos dois tipos de má alimentação que afetam o idoso, a desnutrição desperta mais atenção do que a obesidade, já que pessoas acima de 65 anos tem maior chance de desenvolver desnutrição, em razão de fatores sociais e econômicos, falta de dentes, doenças crônicas, doenças emocionais como a depressão, alterações cognitivas e de paladar, viver sozinho, diminuição ou perda da capacidade funcional, efeitos colaterais de medicamentos entre outros.

Os carboidratos fornecem a maior parte de energia que o organismo necessita para realização de suas atividades diárias, sendo a principal fonte de energia para o cérebro; sua absorção pode ser ligeiramente prejudicada com o avançar da idade, ocasionando resistência à insulina. Conforme explicam Shils et al., (2003), uma forma de solucionar esse problema é ofertar 
ao idoso fontes de carboidrato complexo e fibras solúveis, reduzindo o consumo de açúcar simples. Oferecendo ao idoso a quantidade ideal de carboidratos, entre 55 a $60 \%$ da dieta, o mesmo terá a energia que necessita e serão poupadas as proteínas ingeridas, permitindo que estas realizem sua função de manutenção muscular - a ingestão protética não deverá ultrapassar $15 \%$ da dieta diária, para não sobrecarregar os rins.

Os lipídeos geram energia, formam os hormônios sexuais, participam na solubilidade das vitaminas lipossolúveis (A, D, E, K) e carotenoides, também contribuindo para a manutenção da temperatura corporal. Deve-se limitar seu consumo ao máximo de $30 \%$ da ingestão total e $10 \%$ da ingestão calórica para gorduras saturadas (CUPPARI, 2006; MINISTÉRIO DA SAÚDE, 2006).

Teixeira Neto (2003) ainda discorre sobre a alimentação do idoso, evidenciando que são fundamentais e essenciais nos processos metabólicos também os micronutrientes (vitaminas e minerais), que fazem parte da composição orgânica estrutural; portanto, com o passar da idade, torna-se cada vez mais necessário o consumo de frutas, verduras e legumes, por serem ricas fontes de micronutrientes.

O aporte diário recomendado de micronutrientes é alcançado por meio de uma alimentação variada em todos os grupos alimentares; porém, com a diminuição da absorção de nutrientes, pode ser necessária a suplementação, que deverá ser feita por profissional adequado, como médico ou nutricionista (TIRAPEGUI, 2000).

A fim de evitar a desidratação, deve-se oferecer de 1,5 litro a 2 litros de água diariamente aos idosos, em pequenos volumes e várias vezes ao dia. Aágua transporta nutrientes para o sangue, auxilia no funcionamento de rins, músculos (como o coração, por exemplo), intestino e estômago, além de regular a temperatura corporal e manter a pele saudável, evitando úlceras de pressão (escaras) (MINISTÉRIO DA SAÚDE, 2006; TEIXEIRA NETO, 2003).

Em 2006, o Ministério da Saúde propôs algumas estratégias para favorecer a saúde alimentar do idoso, as quais se resumem em: A) Fazer as refeições em família ou com amigos e em ambiente agradável, evitando isolamento, depressão, discussões e estresse; B) Alimentar-se em períodos regulares, evitando jejuns prolongados com pequenos lanches entre as principais refeições; C) Consumir frutas, verduras e legumes diariamente, variando os tipos; D) Consumir proteína de fonte magra ao menos uma vez ao dia, quatro vezes por semana; E) Consumir alimentos integrais, pois são fontes de fibras e micronutrientes, regulando a função intestinal, as frações de Colesterol e a Glicemia; F) Desestimular o consumo de sal e açúcar à mesa; G) Evitar gorduras animais, preferindo azeite ou óleo vegetal; H) Aumentar o consumo de água para 1,5 litro a 2 litros diários.

\section{Atividade física e envelhecimento}

O envelhecimento é um processo inerente aos seres vivos, porém com perda progressiva das aptidões funcionais do organismo. Esse fato tende a aumentar o risco de sedentarismo, e essas alterações nos campos biopsicossociais colocam em risco a qualidade de vida do idoso, por limitar a capacidade para realizar atividades do cotidiano (ALVES et. al., 2004).

Há alguns anos vem se negligenciando a utilização de exercícios resistidos (com ganho de força e massa óssea) para idosos, por preconceito ou desconhecimento. Porém, a utilização do protocolo adequado na prescrição de exercícios se torna extremamente importante para a qualidade de vida do idoso. Além disso, o fato de exercitar-se promove não só a integração social, como a autonomia do idoso diante das dificuldades do dia a dia.

A massa óssea muda consideravelmente durante as várias etapas da vida. Na infância, adolescência e até os 35 anos de idade, a massa óssea está em constante formação. Nessa etapa, culmina-se no pico da massa óssea e, a partir daí, inicia-se um processo lento de perda óssea, equivalente a 1,5\% ao ano. No início da menopausa, $25 \%$ das mulheres iniciam uma perda bem mais agressiva (de $3 \%$ a $4 \%$ ao ano), passando a apresentar osteoporose (SAMBROOK et al., 2006).

A força muscular máxima é atingida por volta dos 30 anos de idade e se mantém mais ou menos estável 
até a 5. década, quando começa o seu decréscimo. Sabe-se que o exercício de impacto promove o aumento de massa óssea, sendo uma alternativa para idosos os exercícios resistidos.

O objetivo da atividade física é melhorar ao máximo a capacidade funcional do indivíduo. Esse objetivo é alcançado por meio de protocolos de treinamentos que buscam aumentar a capacidade aeróbica, equilíbrio, força muscular e flexibilidade. O declínio do desempenho de força, endurance muscular, velocidade de movimento e potência são notórios em pessoas idosas. Mesmo no envelhecimento natural, se observa significativa perda de força e massa muscular, intimamente ligada à menor atividade física (FREITAS et al., 2006).

Estefani (2007) defende que o aumento da força muscular, promovido pelo treinamento de força, permite aos idosos reverterem situações inesperadas de desequilíbrio. Com a retomada da força e flexibilidade, devolvemos aos idosos movimentos simples, como subir degraus e até mesmo entrar em um veículo sem a ajuda de terceiros, frequentar locais de entretenimento e lazer, contribuindo, portanto, para o aumento na qualidade de vida.

\section{Conclusão}

O envelhecimento é um processo natural ao ser humano, que traz muitas alterações físicas e metabólicas, que podem influenciar o modo de vida dos indivíduos. No Brasil, que sempre foi considerado um país jovem, têm aumentado os índices de envelhecimento, conforme dados do IBGE. Tal fato se deve à queda da mortalidade, avanços na medicina, redução de doenças contagiosas e parasitárias, melhoria na urbanização e saneamento, melhoria de salários e acesso a alimentos antes não consumidos, além da queda de fecundidade, devido à inserção da mulher no mercado de trabalho, aumento da escolaridade e promoção dos métodos de controle da natalidade.

Tão importante quanto ter muitos anos de vida é ter qualidade de vida ao envelhecer. Para ter qualidade de vida, é extremamente importante aliar alimentação saudável com atividade física regular.
Com o aumento da idade, perde-se massa magra e aumenta-se a quantidade de tecido adiposo, o que favorece o ganho de peso quando não há controle alimentar e físico. Nutricionistas e educadores físicos podem colaborar com o aumento da qualidade de vida de idosos, ao adequar as necessidades alimentares e treinos físicos, respectivamente, às mudanças que a idade e estilo de vida exigem, conforme a revisão bibliográfica apresentada.

\section{REFERÊNCIAS}

ABREU, P.F; SESSO, R.C.C; RAMOS, L.R. Aspectos renais no idoso. J. Bras. Nefrol. Escola Paulista de Medicina, Universidade Federal de São Paulo, São Paulo.v.20, n.2, 1998.

ALVES, R. V.; MOTA, J.; COSTA, M. C.; ALVES, J. G. B. Aptidão física relacionada à saúde de idosos: influência da hidroginástica. Rev. Bras. Med. Esporte v. 10 n.1-jan/fev. 2004.

BARBOSAAR; SOUZAJM; LEBRAO ML; LAURENTI R; MARUCCI MFN. Functional limitations of Brazilian elderly by age and gender differences: data from SABE survey. Cad. Saúde Publica, v. 2, p. 1177-85, 2005.

\section{BARRETO, P.M.S. Perfil alimentar de idosos} participantes de grupos de terceira idade no município de Araraquara, SP. 2004. 138f. Dissertação (Mestrado em Ciências Nutricionais) Faculdade de Ciências Farmacêuticas, UNESP, Araraquara, 2004.

\section{BRASIL. MINISTÉRIO DA SAÚDE. Secretaria} de Atenção à Saúde. Departamento de Atenção Básica. Alimentação saudável para a pessoa idosa: um manual para profissionais de saúde / Ministério da saúde, Secretaria de Atenção à Saúde, Departamento de Atenção Básica. Brasília: Editora do Ministério da Saúde, 2009. 
CAETANO, J.A; COSTA, A.C; SANTOS, Z.M.S.A; SOARES. E. Descrição dos fatores de risco para alterações cardiovasculares em um grupo de idosos, 2008. Florianópolis, v.17. n.2, p. 327-35, abr-jun. 2008.

CAMPOS, M. T.F. S; MONTEIRO, J.B. R; ORNELAS, A.P.R.C. Fatores que afetam o consumo alimentar e a nutrição do idoso, Rev. Nutr., Campinas, v.13. n.3 p. 157-165, set.,/dez., 2000.

CARVALHO, J.A.M.; GARCIA, R.A. O envelhecimento da população brasileira: um enfoque demográfico. Cad. Saúde Pública, Rio de Janeiro, v. 19, n.3, jun. 2003.

CRESS, M.E., BUCHNER, D.M. E QUESTAD, K.A. Exercise: effects on physical functional performance in independent older adults. Journal of Gerontology Advanced Biological Sciences Medical and Science, v.54, n.5, p.242 - 248, 1999.

CUPPARI, L. Guia de Nutrição. Nutrição do Adulto. 2. Ed. São Paulo: Manole, 2006.

ESTEFANI, G. A. Perfil de idosos atendidos em ambulatório de geriatria segundo a ocorrência de quedas. 2007. 118f. Dissertação (Mestrado em Gerontologia) - Universidade Estadual de Campinas, Faculdade de Educação. Programa de Pós-Graduação em Gerontologia, Campinas, 2007.

FERNANDES, M.P. et al. Trabalho feminino e diminuição da taxa de fecundidade no Brasil nos últimos 50 anos. Rev.Saúde Coletiva, v. 49 n 8 p. 71-76, 2011.

FREITAS, E.V.; KOPILLIER, D. Atividade Física no Idoso. In: Freitas E.V, et al. Tratado de Geriatria e Gerontologia. 2. ${ }^{a}$ ed. Rio de Janeiro: Guanabara Koogan, 2006. cap.58, p.586-59.
INSTITUTO BRASILEIRO DE GEOGRAFIAE ESTATISTICA-IBGE. Disponível em: http//: www.ibge.gov.br/home/presidencia/noticias/ noticiavisualiza.php?id_noticia. Acesso em 13/dez. / 2012.

OLIVEIRA, D.A.A.P; GOMES, L; OLIVEIRA, R. Prevalência de depressão em idosos que frequentam centros de convivência. Revista de Saúde Publica, São Paulo, v.40 n.4, ago. 2006.

MAHAN, L.K; SCOTT-STUMP, S. Krause, alimentos, nutrição e dietoterapia, 10 ed. São Paulo: Roca, 2001.

MATSUDO S. M. ; MATSUDO, V.K.R.; BARROS NETOT.L. Impacto do envelhecimento nas variáveis antropométricas, neuromotoras e metabólicas da aptidão física. Rev.Bras Cien Mov. v. 8 n. 4 p. $21-32$, set. 2000.

MENDES, M.R.S.S.; GUSMÃO, J.L; FARO, A.C.M.F; LEITE, R.C.B.O. A situação social do idoso no Brasil: uma breve consideração, 2005. Acta Paul. Enferm. v.18, n.4 oct./dec. 2005.

MENEZES, T.N.; MARUCCI, M.F.N. Antropometria de idosos residentes em instituições geriátricas, Fortaleza, Rev. Saúde Publ. v.39 n.2 p.169-175, 2005.

MINISTÉRIO DA SAÚDE, Secretaria de Vigilância em Saúde e Secretaria de Atenção à Saúde - Programa Nacional de DST e AIDS. Alimentação e Nutrição para Pessoas Vivendo com HIV e AIDS. Brasília: Ministério da Saúde, 2006.

MORIGUTI, J. C.; IUCIF JÚNIOR, N.; FERRIOLI, E. Nutrição no idoso. In: DUTRA-DEOLIVEIRA, J. E.; MARCHINI, J. S. Ciências nutricionais. São Paulo: Sarvier, 1998. Cap 14.p.239-251. 
NAJAS, M.; YAMATTO, T. H. Nutrição na Maturidade. In: Nestlé Nutrition: Avaliação do estado nutricional de idosos. São Paulo, 2008.

NOBREGA, A.C.L. et al. Posicionamento oficial da Sociedade Brasileira de Medicina do Esporte e da Sociedade Brasileira de Geriatria e Gerontologia: atividade física e saúde no idoso. Rev. Brasileira de Medicina do Esporte, Niterói, v.5.n.6. nov/ dec., 1999.

PIERINI, D.T; NICOLA, M; OLIVEIRA, E.P. Sarcopneia: alterações metabólicas e conseqüências no envelhecimento, 2009. Rev. bras.Ci. e Mov, v.17n.3.p.96-103, 2009

RIBEIRO, L.C.C.C; ALVES, P.B; MEIRA, E.P. Percepção dos idosos sobre as alterações fisiológicas do envelhecimento. Cienc. Cuid. Saude, abr/jun; v.8 n.2 p.220-227, 2009.

RIBEIRO, S. M. L. ; J Tirapegui . Nutrição e envelhecimento. In: Julio Tirapegui.. (Org.).

Nutrição fundamentos e aspectos atuais. São Paulo: Atheneu, 2000, v. 1, p. 127-140.

ROSSI, E. Envelhecimento do sistema osteoarticular. Rev. Einstein. v. 6, Supl. 1: S7S12. 2008.

RUIVO, S; VIANA, P; MARTINS, C; BAETA,C. Efeito do envelhecimento cronológico na função pulmonar. Comparação da função respiratória entre adultos e idosos saudáveis. Rev. Port Pneumol.
Lisboa, v.15, n.4, ago. 2009.

SAMBROOK, P.; COOPER C. Osteoporosis. Pathogenesis, diagnosis and treatment in older alduts. Rheum Dis Clin North Am. v.26, n.3. p.569-91, 2006.

\section{SHILS, E. M. ET AL. Tratado de Nutrição} Moderna na Saúde e na Doença. 9. ed. São Paulo: Manole, 2003.

\section{SOARES J; ALABERSE, S. Envelhecimento e} Atividade Física. IN: RAMOS L. R, TANIOLO J. NETO. Guia de Geriatria e Gerontologia. Barueri: Manole, 2005. cap.20, p.255-70.

TEIXEIRA NETO, F. Nutrição Clínica. 1 Ed. Rio de Janeiro. Guanabara Koogan, 2003. CUNHA, S. F. C. ; CUNHA, D. F. . Nutrição em geriatria. IN: FAUSTINO TEIXEIRANETO. (Org.). Nutrição Clínica. Rio de Janeiro: Guanabara Koogan, 2003, Cap. 5.p. 279-290.

TIRAPEGUI, J. Nutrição: Fundamentos e Aspectos Atuais. São Paulo: Atheneu, 2000. v.1 Cap.10.

VERAS, R. Envelhecimento populacional contemporâneo: demandas, desafios e inovações. Rev. Saúde. Pública, n.43 v.3, p.548-54, 2009.

WONG, L.L.R.; CARVALHO, J.A. O rápido processo de envelhecimento populacional do Brasil: sérios desafios para as políticas públicas. Rev. bras. Est. Pop., São Paulo, v. 23, n. 1, p. 5-26, jan./jun. 2006.

RECEBIDO EM 1/8/2013

ACEITO EM 27/9/2013 